\title{
Terminal seizure frequency and its relation to SUDEP
}

\author{
Deng Chen ${ }^{1}$, Yang $\mathrm{Si}^{2}$, Jun $\mathrm{He}^{3}$, Ying Deng ${ }^{3}$, Tao $\mathrm{Chen}^{4}$, Yu-jin $\mathrm{He}^{3}$, Ling $\mathrm{Liu}^{1 *}$, Dong Zhou ${ }^{1 *}$
}

1. Department of Neurology, West China Hospital, Sichuan University, Wai Nan Guo Xue Lane 37\#, Chengdu 610041, China

2. Department of Neurology, Sichuan Provincial People's Hospital, Chengdu, Sichuan, China

3. Sichuan Center of Disease Control and Prevention, Sichuan, Chengdu 610041,China

4. Department of Neurology, Chengdu 416 Hospital, Chengdu, Sichuan, China

*. Corresponding Authors, Department of Neurology, West China Hospital, Sichuan University, Wai Nan Guo Xue Lane 37\#, Chengdu 610041, China, Email: Ling Liu: zjllxx1968@163.com; Dong Zhou: zhoudong66@yahoo.de

\begin{abstract}
Background: Sudden unexpected death in epilepsy (SUDEP) is a major cause of death in epilepsy patients. Several risk factors have been implicated, including early age of onset, tonic-clonic seizures and antiepileptic drugs. However, whether SUDEP patients have a greater frequency of seizures in the few months before death is unclear. We investigated the end-time seizure frequency and its relation to SUDEP from a large group of tonic-clonic epilepsy patients in rural West China.

Methods: We used the database from the Convulsive Epilepsy Control and Management Program in West China, which use phenobarbital (PB) as a treatment for convulsive epilepsy. Probable SUDEP patients were included according to pre-set criteria. A verbal autopsy was undertaken for each case. By matching each patient's age, sex, date of joining the program, time on follow-up and baseline seizure frequency, we set up a 1:5 ratio control group. SPSS 21.0 statistics were applied to compare the differences on seizure frequency 3 months prior to SUDEP between probable SUDEP and controls. Furthermore, the dynamic changing of terminal seizure frequency 6-9 months, 3-6 months and 3 months prior to SUDEP was also analyzed.

Results: A total of 41 probable SUDEP sufferers were identified out of 7844 patients during 10 years of follow-up. The SUDEP group had a significantly higher tonic-clonic seizure frequency 3 months before their deaths than the control group $(p=0.023)$. At the same time, their seizure-free rate was lower than the control group $(\mathrm{p}=0.025)$. Probable SUDEP patients who were followed
\end{abstract}


up over 12 months were set as a subgroup. They had more tonic-clonic seizures 3 months prior to death compared to the control group $(\mathrm{p}=0.010)$. They also had a increasing on seizure frequency in their terminal phase ( 3 months prior) comparing to earlier stage $(3-6$ months prior) $(\mathrm{p}=0.029)$. Furthermore, the end-time PB dose in the SUDEP group was higher than the control group $(\mathrm{p}=$ 0.002).

Conclusion: SUDEP patients had more frequent tonic-clonic seizures 3 months before their deaths. Higher seizure frequency increases the exposure to peri-ictal pathophysiological events, which possibly relate to SUDEP. This phenomenon may be due to the drug resistance potential of these patients or the high dose of PB. Further research is required to ascertain the underlying mechanism of SUDEP.

Keywords: sudden unexpected death in epilepsy, SUDEP, seizure frequency

\section{Background}

Sudden unexpected death in epilepsy (SUDEP) is one of the major causes of death in patients with epilepsy[1, 2]. SUDEP was firstly defined by Nashef[3]. In different studies, the incidence of SUDEP was reported ranging from only 0.35 to 9.3 per 1000 person-years [4, 5]. The current understanding of SUDEP mostly comes from a series of cases or case control studies[6]. These studies managed to identify several risk factors for SUDEP[4], in which increased frequency of seizures was most commonly reported[4, 5, 7, 8].

Seizure frequency was most often measured by actual seizure counts annually or monthly. In one large cohort study by Nilsson on epidemiology for SUDEP[9], patients having seizures over one observational year had a 23 times higher relative risk for SUDEP than patients without seizures. A more detailed analysis on seizure types revealed that tonic-clonic seizures significantly increased the risk[4]. These studies were somehow inconsistent with each other on the extent of the increased risks, but this could be explained by the different designs of the studies[5]. In general, high seizure frequency, especially tonic-clonic seizures, was regarded as the strongest risk factor$[4,5,10]$.

However, the distribution of seizures according to time was not determined in these studies. The dynamic change in seizure frequency was not sufficiently reported. In 2014, a community-based 
study in Cornwall, UK[11], reported a phenomenon where 91\% of probable SUDEP patients had increased seizure frequency or seizure deterioration 3-6 months prior to their deaths. Our research team published an article in 2016 on the risk factors for probable SUDEP[12], which concluded that non-seizure-free patients were at an increased risk for SUDEP, especially in the months before their deaths. These results implied that SUDEP patients may have more seizures before their deaths. We report a case control study in rural West China in order to clarify the relation between end-time seizure frequency and SUDEP.

\section{Methods}

This study was approved by the Sichuan University Ethical Standards Committee on Human Experimentation.

All patient data were obtained from the database of the Convulsive Epilepsy Control and Management Program in West China[13, 14], which focused on monitoring epilepsy epidemiology and treating patients with phenobarbital (PB) under guidance. The program included 16 centres in rural West China from May 2005 to December 2015 and covered more than 10,000 epilepsy patients. The inclusion criteria for this program were published in our prior report[13]. Patients were diagnosed by primary healthcare physicians and confirmed by referral neurologists according to the International League Against Epilepsy definition of generalised tonic-clonic epilepsy[15]. They were given PB as an initial treatment at a dose of $60 \mathrm{mg} / \mathrm{d}$, which was adjusted according to their status of seizure control. Continuous monthly follow-ups were conducted by primary healthcare physicians. Data were collected annually by local Centers for Disease Control and Prevention (CDC) and further processed by this research team.

The criteria for probable SUDEP were:

(1). Witnessed or unwitnessed death of patients while they were in a reasonable state of health;

(2). Patients who were in relatively safe circumstances and performing normal activities;

(3). No evidence on the cause of death according to autopsy or verbal autopsy, including status epilepticus or death caused by a particular seizure directly;

4. Sudden death with no obvious symptoms or signs of approaching death at the last follow-up. A verbal autopsy[16] was performed for each eligible case by directly contacting the first witness and gathering information from the local CDC and hospital. One unified autopsy form was applied 
for quality control as our previous paper reported.

For each probable SUDEP case included, we assigned five controls from the database by matching age ( \pm 5 years), sex, date of joining the program (same year), time on follow-up, and baseline seizure frequency (It is done by selecting the 5 closest cases on seizure frequency according to the baseline data collected when they joined the program). The follow-up records of each case and its controls were extracted from the database of the program and merged into two groups: the probable SUDEP group and the control group.

SPSS 21.0 was applied for statistical analysis. Qualitative data were compared in a chi-square test. For independent measurement samples, data in normal distribution were compared on the Student's t-test, and other forms of distributions were examined by the Mann-Whitney U test. Pairing samples were also analysed by either the matched-pair t-test or the Wilcoxon matched-pairs signed-rank test, according to their distribution. In addition, data in normal distributions were demonstrated in mean and variance; other forms of data were given by quartile. We assigned $\alpha=0.05$ as a degree of inspection, with $\mathrm{p}<0.05$ considered as statistically significant.

We focused on comparing seizure frequency at different times in both groups. A subgroup of follow-up lasting for more than 12 months was designed to analyse the dynamic changes in each patient's seizure frequency.

\section{Results}

Since 2005, a total of 7844 patients with convulsive epilepsy have been enrolled in the project with a total follow-up person-years of 23739.58 , from which 41 probable SUDEP cases were reported, making the incidence of SUDEP 1.72\% every person-year. Each probable SUDEP was assigned with five control cases, as described above. The baseline characteristics of the two cohorts are listed in Table 1.

The program was successful in controlling tonic-clonic seizures. In both groups, the seizure counts were lower than baseline after treatments (Mann-Whitney U test, SUDEP group: $p<0.001$; control group: $\mathrm{p}<0.001$ ).

The annual seizure frequency was designed to measure the severity of the epilepsy. A 
Kolmogorov-Smirnov test was applied to the data of both cohorts, but no normal distribution fitted either one. Thus, the Mann-Whitney U test was applied for this calculation. The average annual seizure frequency of the SUDEP group was $6.53 / \mathrm{y}$, which was higher than the control group $(3.96 / y)$, but was statistically insignificant $(\mathrm{p}=0.59)$.

Table 1: Baseline Characteristics

\begin{tabular}{|llll|}
\hline & Probable SUDEP & Control & $\mathrm{P}$ value \\
\hline Sex & 19 male $(46.34 \%)$ & 95 male $(46.34 \%)$ & $\mathrm{P}=1.00^{*}$ \\
Age & $40.61 \pm 15.39 \mathrm{y}$ & $40.08 \pm 15.47 \mathrm{y}$ & $\mathrm{P}=0.84^{*}$ \\
Height & $157.39 \pm 8.28 \mathrm{~cm}$ & $158.70 \pm 8.76 \mathrm{~cm}$ & $\mathrm{P}=0.38^{*}$ \\
Weight & $54.24 \pm 9.21 \mathrm{~kg}$ & $52.98 \pm 11.46 \mathrm{~kg}$ & $\mathrm{P}=0.55^{*}$ \\
Baseline tonic-clonic Seizure Frequency & $8.10 \pm 10.83 / \mathrm{y}$ & $6.07 \pm 9.43 / \mathrm{y}$ & $\mathrm{P}=0.32^{*}$ \\
Baseline dosage of PB & $84.88 \pm 34.14 \mathrm{mg}$ & $76.52 \pm 28.60 \mathrm{mg}$ & $\mathrm{P}=0.13^{*}$ \\
Mean duration of epilepsy & $20.64 \pm 15.28 \mathrm{y}$ & $20.20 \pm 12.69 \mathrm{y}$ & $\mathrm{P}=0.82^{*}$ \\
Age of onset & $20.43 \pm 14.57 \mathrm{y}$ & $22.67 \pm 16.68 \mathrm{y}$ & $\mathrm{P}=0.44^{*}$ \\
Combined medication & $6(14.63 \%)$ & $27(13.17 \%)$ & $\mathrm{P}=0.88^{*}$ \\
\hline
\end{tabular}

${ }^{*}$ Chi-square test

* Student's t test

To be seizure-free for 3 months before death was counted as an index for seizure remission. In this study, 19 out of 41 probable SUDEP patients were reported as seizure-free for 3 months before they died; in the control group, 155 patients were seizure-free over the same time span. A chi-square test indicated that the SUDEP group had significantly higher non-seizure-free incidences than the control group $(\mathrm{p}<0.001)$. Another comparison on actual seizure counts for the 3 months before death between the two groups showed that the SUDEP group had more seizures before they died than the control group (Mann-Whitney $U$ test, $p=0.023$ ).

Another interesting finding was the shift of dosage on PB. With comparable initial doses of PB, the SUDEP group had a significant increase in PB dosage from baseline until their deaths (Mann-Whitney U test, $\mathrm{p}=0.020$ ). However, at the same time, the control group certainly had an increase in dosage but clearly not enough to make a difference (Mann-Whitney U test, $\mathrm{p}=0.13$ ). The PB doses in the end were also higher in the probable SUDEP group $(p=0.0020)$.

A subgroup analysis for patient follow-ups for more than 12 months $(\mathrm{n}=35)$ exposed a trend of seizure deterioration within 3 months. The Mann-Whitney U test on seizure counts for 1-3 months, 3-6 months and 6-9 months between the SUDEP group and the control group was $p=0.010, p=$ 
0.62 and $p=0.64$, respectively. The Wilcoxon matched-pairs signed-rank test showed that seizure counts significantly increased 1-3 months before death compared to $3-6$ months $(p=0.020)$. However, there was no difference 3-6 months and 6-9 months before death.

\section{Discussion}

The concept of SUDEP has been recognized for a number of decades. It reminds neurologists that epilepsy is not a benign disease. Recent researches have made great advances on understanding this fatal condition. The increasing cohort studies have proven several risk factors, among which is tonic-clonic seizure frequency. However, these studies focused on overall seizure frequency and did not analyse it according to the different time points of the disease course. This leaves the question of whether tonic-clonic seizures became more frequent right before the patients died. The research group generated a cohort from a large, long-term follow-up program by matching baseline characteristics, including initial seizure frequency, in an effort to reveal the relation between terminal seizure frequency and SUDEP.

In this study, both the SUDEP group and the control group responded to treatment with PB, and there was no statistical difference in annual seizure frequency. This result seemed to be inconsistent with many other studies[8, 9, 11, 17-19]. However, it could be explained by the matching of initial seizure frequencies, which were not covered by those earlier studies. In addition, one of our previous reports[12] with a different design compared the seizure frequency of the SUDEP group, proving tonic-clonic seizures were more frequent in that group than in other epilepsy patients.

Table 2: Key findings in this research

\begin{tabular}{|llll|}
\hline & Probable SUDEP & Control & $\mathrm{P}$ value \\
\hline Average annual seizure frequency & $4.138(0.356,7.900) / \mathrm{y}$ & $1.5(0,5.547) / \mathrm{y}$ & $\mathrm{P}=0.59^{*}$ \\
Seizure frequency 3 months prior to death & $0(0,10) / \mathrm{y}$ & $0(0,0) / \mathrm{y}$ & $\mathrm{P}=0.023^{*}$ \\
Seizure free 3 months prior to death & 19 & 155 & $\mathrm{P}<0.001^{*}$ \\
PB dosage prior to death & $90 \mathrm{mg}(90 \mathrm{mg}, 165 \mathrm{mg})$ & $90 \mathrm{mg}(60 \mathrm{mg}, 120 \mathrm{mg})$ & $\mathrm{P}=0.002^{*}$ \\
Poor Complience & 7 & 19 & $\mathrm{P}=0.16^{*}$ \\
Combined medication & 4 & 17 & $\mathrm{P}=0.77^{*}$ \\
\hline
\end{tabular}

\footnotetext{
* Mann-Whitney U test
}

${ }^{*}$ Chi-square test 
Other than a general comparison, this study focused on more specific time periods before SUDEP.

We manually set 3 months before death as our cut-off, and compared the seizure frequencies. Interestingly, even after PB treatment, probable SUDEP patients seemed to have more tonic-clonic seizures and less seizure-free rates by the time of death than the control group. Also, a subgroup analysis was done by including those SUDEP patients who had a follow-up time for longer than 12 months, comparing seizure counts $1-3$ months, 3-6 months and 6-9 months before death. We found that for SUDEP patients, tonic-clonic seizures were more frequent during the first 3-month period. No difference was shown between the two subgroups on 3-6 months and 6-9 months seizure frequency.

The increased tonic-clonic seizure counts prior to patient deaths raised two important questions:

(1) What is the role of seizure frequency in SUDEP?

(2) What is the cause of this sudden deterioration?

The mechanism underlying SUDEP is not clear[20]; however, recent studies have provided us with some clues. Current hypotheses focus on three possible factors: respiratory dysfunction[21-23], cardiac dysfunction[24, 25] and post-ictal arousal deficits[5, 26]. The rising count of seizures increases the patients' chance of being exposed to these factors and may trigger a series of life-threatening events, which are distinguished from the usual type of seizures. However, this theory does not explain the situation of those patients who were seizure-free, which was rare, but still suffered from SUDEP.

In this study, we did not find a reasonable explanation on the cause of the deterioration of seizures. Suspected reasons include protocol violation, alcohol abuse, drug abuse, shifting medication and combination of antiepileptic drugs $[5,10]$. However, none of these reasons was different between the probable SUDEP group and the control group except the PB dose. Even though initial PB doses were comparable, the end-time (3-month) PB dose in the SUDEP group was significantly higher than their own baseline and the control group.

Since our program aimed at seizure control, the dose of PB was increased on the condition that tonic-clonic seizures were not well controlled. This difference in seizure counts might indicate a potentially refractory trend in the SUDEP group on PB treatment. Also, there is a possibility that a high dose of PB worked as a risk factor for SUDEP. PB is a first-generation antiepileptic drug, which enhances the influx of chloride ions and inhibits the excitability of neurons[27]. No 
previous report has shown sufficient evidence[7, 28-31] on whether other antiepileptic drugs, including PB, increased SUDEP risks, much less their relation to the dosage. Thus, more evidence is needed to clarify the relation between SUDEP and antiepileptic drugs.

There are some limitations of this study. First, matching controls, other than randomly assigning them, brought about selection bias. Second, the follow-up time among deceased cases varied significantly, where the longest was 61 months and the shortest was only 3 months. Third, this program only gave $\mathrm{PB}$ as a treatment. Although patients have the right to take other antiepileptic drugs, such as carbamazepine, we cannot ignore the possibility that these patients were not treated individually, or to put it more simply, not in the best way. Thus, perhaps they would respond well to the treatment of other antiepileptic drugs. Further research is required to examine these conclusions under stricter conditions.

\section{Conclusions}

Tonic-clonic seizures were more frequent in SUDEP patients right before their deaths (possibly up to 3 months before death). This increased frequency raises the chance of patient exposure to peri-ictal pathophysiological factors and may contribute to SUDEP. The cause of this phenomenon is still unclear. The increased frequency might be explained by the drug-resistant potential of these patients. In addition, the high dose of phenobarbital could be a risk factor for SUDEP. Further research of large scale, community-based, long-term follow-up and a strict time limit is still needed to explore the cause of SUDEP.

\section{Acknowledgement}

We would like to thank all of the local physicians and health care providers for their efforts on continually reporting of latest data. We also appreciate the help from Central Disease Control on running this program.

\section{Disclosure}

This study was funded by the Chinese Ministry of Health and the Sichuan Province Health Bureau. It was supported by National Science Foundation of China (NSFC), No. 81420108014 and No. 81371529. All the authors report no more conflict of interest. 
[1] Mu J, Liu L, Zhang Q, Si Y, Hu J, Fang J, Gao Y, He J, Li S, Wang W, Wu J, Sander JW, Zhou D. Causes of death among people with convulsive epilepsy in rural West China: a prospective study. Neurology 12011;77: 132-7.

[2] Si Y, Chen D, Tian L, Mu J, Chen T, Liu L, Deng Y, He J, Li Y, He L, Zhou D. Update on causes of premature death in people with convulsive epilepsy in rural West China. Epilepsia 12016;57: e117-20.

[3] Nashef L. Sudden unexpected death in epilepsy: terminology and definitions. Epilepsia 11997;38: S6-8.

[4] Tomson T, Walczak T, Sillanpaa M, Sander JW. Sudden unexpected death in epilepsy: a review of incidence and risk factors. Epilepsia 12005;46 Suppl 11: 54-61.

[5] Devinsky O, Hesdorffer DC, Thurman DJ, Lhatoo S, Richerson G. Sudden unexpected death in epilepsy: epidemiology, mechanisms, and prevention. Lancet Neurol 12016;15: 1075-88.

[6] Tomson T, Nashef L, Ryvlin P. Sudden unexpected death in epilepsy: current knowledge and future directions. Lancet Neurol 12008;7: 1021-31.

[7] Hesdorffer DC, Tomson T, Benn E, Sander JW, Nilsson L, Langan Y, Walczak TS, Beghi E, Brodie MJ, Hauser WA, Epidemiology ICo. Do antiepileptic drugs or generalized tonic-clonic seizure frequency increase SUDEP risk? A combined analysis. Epilepsia 12012;53: 249-52.

[8] Ryu HU, Hong JP, Han SH, Choi EJ, Song JH, Lee SA, Kang JK. Seizure Frequencies and Number of Anti-epileptic Drugs as Risk Factors for Sudden Unexpected Death in Epilepsy. J Korean Med Sci 12015;30: 788-92.

[9] Nilsson L, Farahmand BY, Persson PG, Thiblin I, Tomson T. Risk factors for sudden unexpected death in epilepsy: a case-control study. Lancet 11999;353: 888-93.

[10] Hesdorffer DC, Tomson T, Benn E, Sander JW, Nilsson L, Langan Y, Walczak TS, Beghi E, Brodie MJ, Hauser A, Epidemiology ICo, Subcommission on M. Combined analysis of risk factors for SUDEP. Epilepsia 12011;52: 1150-9.

[11] Shankar R, Jalihal V, Walker M, Laugharne R, McLean B, Carlyon E, Hanna J, Brown S, Jory C, Tripp M, Pace A, Cox D, Brown S. A community study in Cornwall UK of sudden unexpected death in epilepsy (SUDEP) in a 9-year population sample. Seizure 12014;23: 382-5.

[12] Zhang WW, Si Y, Chen T, Chen D, Liu L, Deng Y, He J, Li Y, Zhou D. Risks of probable SUDEP among people with convulsive epilepsy in rural West China. Seizure 12016;39: 19-23.

[13] Liu L, Zhang Q, Yao Z, Wang X, Zhu C, Gao Y, He J, Wang Z, Zhang N, D'Souza W, Zhou D. The operational model of a network for managing patients with convulsive epilepsy in rural West China. Epilepsy Behav 12010;17: 75-81.

[14] Hu J, Si Y, Zhou D, Mu J, Li J, Liu L, Zhu CR, Deng Y, He J, Zhang NM, Chen XF. Prevalence and treatment gap of active convulsive epilepsy: a large community-based survey in rural West China. Seizure 12014;23: 333-7.

[15] Proposal for revised clinical and electroencephalographic classification of epileptic seizures. From the Commission on Classification and Terminology of the International League Against Epilepsy. Epilepsia 11981;22: 489-501.

[16] Lathers CM, Schraeder PL. Verbal autopsies and SUDEP. Epilepsy Behav 12009;14: 573-6.

[17] Leestma JE, Walczak T, Hughes JR, Kalelkar MB, Teas SS. A prospective study on sudden unexpected death in epilepsy. Ann Neurol 11989;26: 195-203.

[18] Ficker DM, So EL, Shen WK, Annegers JF, O'Brien PC, Cascino GD, Belau PG. 
Population-based study of the incidence of sudden unexplained death in epilepsy. Neurology 11998;51: $1270-4$.

[19] Lhatoo SD, Langan Y, MacDonald BK, Zeidan S, Sander JW. Sudden unexpected death: a rare event in a large community based prospective cohort with newly diagnosed epilepsy and high remission rates. J Neurol Neurosurg Psychiatry 11999;66: 692-3.

[20] Massey CA, Sowers LP, Dlouhy BJ, Richerson GB. Mechanisms of sudden unexpected death in epilepsy: the pathway to prevention. Nat Rev Neurol 12014;10: 271-82.

[21] Watanabe K, Hara K, Hakamada S, Negoro T, Sugiura M, Matsumoto A, Maehara M. Seizures with apnea in children. Pediatrics 11982;70: 87-90.

[22] Nashef L, Walker F, Allen P, Sander JW, Shorvon SD, Fish DR. Apnoea and bradycardia during epileptic seizures: relation to sudden death in epilepsy. J Neurol Neurosurg Psychiatry 11996;60: 297-300

[23] Bateman LM, Li CS, Seyal M. Ictal hypoxemia in localization-related epilepsy: analysis of incidence, severity and risk factors. Brain 12008;131: 3239-45.

[24] Ryvlin P, Nashef L, Lhatoo SD, Bateman LM, Bird J, Bleasel A, Boon P, Crespel A, Dworetzky BA, Hogenhaven H, Lerche H, Maillard L, Malter MP, Marchal C, Murthy JM, Nitsche M, Pataraia E, Rabben T, Rheims S, Sadzot B, Schulze-Bonhage A, Seyal M, So EL, Spitz M, Szucs A, Tan M, Tao

JX, Tomson T. Incidence and mechanisms of cardiorespiratory arrests in epilepsy monitoring units (MORTEMUS): a retrospective study. Lancet Neurol 12013;12: 966-77.

[25] Rocamora R, Kurthen M, Lickfett L, Von Oertzen J, Elger CE. Cardiac asystole in epilepsy: clinical and neurophysiologic features. Epilepsia 12003;44: 179-85.

[26] Blumenfeld H. Impaired consciousness in epilepsy. Lancet Neurol 12012;11: 814-26.

[27] Polc P. Enhancement of GABAergic inhibition: a mechanism of action of benzodiazepines, phenobarbital, valproate and L-cycloserine in the cat spinal cord. Electroencephalogr Clin Neurophysiol Suppl 11982;36: 188-98.

[28] Tomson T, Hirsch LJ, Friedman D, Bester N, Hammer A, Irizarry M, Ishihara L, Krishen A, Spaulding T, Wamil A, Leadbetter R. Sudden unexpected death in epilepsy in lamotrigine randomized-controlled trials. Epilepsia 12013;54: 135-40.

[29] Lukic S, Bjelakovic B, Spasic M. Causal or casual association between lamotrigine and sudden unexpected death in females with epilepsy? Epilepsia 12012;53: 762-3.

[30] Aurlien D, Larsen JP, Gjerstad L, Tauboll E. Increased risk of sudden unexpected death in epilepsy in females using lamotrigine: a nested, case-control study. Epilepsia 12012;53: 258-66.

[31] Timmings PL. Sudden unexpected death in epilepsy: is carbamazepine implicated? Seizure 11998;7: 289-91. 